Research paper

\title{
Plasma kallikrein enhances platelet aggregation response by subthreshold doses of ADP
}

\author{
Tatiana F. Ottaiano a, Sheila S. Andrade b, d, Cleide de Oliveira a, Mariana C.C. Silva a , \\ Marcus V. Buri ${ }^{\text {c }}$, Maria A. Juliano ${ }^{\text {c }}$, Manoel J.B.C. Girão ${ }^{\text {b, d }}$, Misako U. Sampaio a , \\ Alvin H. Schmaier ${ }^{\mathrm{e}}$, Alexander Wlodawer ${ }^{\mathrm{f}}$, Francisco H.A. Maffei ${ }^{\mathrm{g}}$, \\ Maria Luiza V. Oliva ${ }^{\text {a, * }}$
}

a Department of Biochemistry, Universidade Federal de São Paulo, São Paulo 04044-020, Brazil

b Department of Gynecology, Universidade Federal de São Paulo, São Paulo 04024-002, Brazil

${ }^{\mathrm{c}}$ Department of Biophysics, Universidade Federal de São Paulo, São Paulo 04044-020, Brazil

d Charitable Association of Blood Collection - COLSAN São Paulo, SP, Brazil

e Case Western Reserve University and University Hospitals Cleveland Medical Center, Cleveland, OH, USA

${ }^{\mathrm{f}}$ Macromolecular Crystallography Laboratory, Center for Cancer Research, National Cancer Institute, Frederick, MD, USA

${ }^{g}$ Department of Orthopedics and Surgery, Universidade Estadual Paulista, Botucatu, Brazil

\section{A R T I C L E I N F O}

\section{Article history:}

Received 23 September 2016

Received in revised form

16 December 2016

Accepted 18 January 2017

Available online 20 January 2017

\section{Keywords:}

ADP

Integrin alphallbbeta3

Plasma kallikrein

Platelet aggregation

\begin{abstract}
A B S T R A C T
Human plasma kallikrein (huPK) potentiates platelet responses to subthreshold doses of ADP, although huPK itself, does not induce platelet aggregation. In the present investigation, we observe that huPK pretreatment of platelets potentiates ADP-induced platelet activation by prior proteolysis of the Gprotein-coupled receptor PAR-1. The potentiation of ADP-induced platelet activation by huPK is mediated by the integrin $\alpha_{\mathrm{IIb}} \beta_{3}$ through interactions with the KGD/KGE sequence motif in huPK. Integrin $\alpha_{\mathrm{IIb}} \beta_{3}$ is a cofactor for huPK binding to platelets to support PAR-1 hydrolysis that contributes to activation of the ADP signaling pathway. This activation pathway leads to phosphorylation of Src, AktS ${ }^{473}$, ERK1/2, and p38 MAPK, and to $\mathrm{Ca}^{2+}$ release. The effect of huPK is blocked by specific antagonists of PAR-1 (SCH 19197) and $\alpha_{\mathrm{IIb}} \beta_{3}$ (abciximab) and by synthetic peptides comprising the KGD and KGE sequence motifs of huPK. Further, recombinant plasma kallikrein inhibitor, rBbKI, also blocks this entire mechanism. These results suggest a new function for huPK. Formation of plasma kallikrein lowers the threshold for ADP-induced platelet activation. The present observations are consistent with the notion that plasma kallikrein promotes vascular disease and thrombosis in the intravascular compartment and its inhibition may ameliorate cardiovascular disease and thrombosis.
\end{abstract}

๑) 2017 Elsevier B.V. and Société Française de Biochimie et Biologie Moléculaire (SFBBM). All rights reserved.

\section{Introduction}

The serine proteinase human plasma kallikrein (huPK, EC 3.4.21.34) acts in inflammation and the intrinsic blood coagulation system $[1,2]$ and is generated following activation of plasma prekallikrein (PPK) by factor XIIa or prolylcarboxypeptidase. HuPK is a 86-88 kDa glycoprotein that circulates in plasma in a concentration of $20-50 \mu \mathrm{g} / \mathrm{mL}(\sim 0.5 \mu \mathrm{M})$ in complex with high molecular

\footnotetext{
* Corresponding author. Department of Biochemistry, São Paulo Federal University, Rua Três de maio, 100, São Paulo CEP 04044-020, Brazil.

E-mail address: olivaml.bioq@epm.br (M.L.V. Oliva).
}

weight kininogen (HK), its primary cofactor and substrate [3-5]. A pleiotropic enzyme, huPK generates bradykinin (BK) from HK [6] and participates in the initiation of the contact system activation amplifying FXII activation leading to FXIIa and, subsequently, FXI activation leading to thrombin and fibrin formation $[1,6]$. HuPK regulates vascular responses in conditions such as diabetes and is associated with accelerated cardiovascular diseases [7-9].

In previous studies, the effect of huPK on platelet aggregation was investigated [10]. Although huPK itself does not induce platelet aggregation, Cassaro et al. [10] show that huPK potentiates aggregation induced by low doses of ADP. Like trypsin huPK cleaves internal Arg or Lys bonds, activating PAR-1 following cleavage of Arg41 but does not induce platelet aggregation through PAR-1 
cleavage [7].

This study shows that huPK sensitizes human platelets by 1) binding to platelet integrins through its KGD and KGE motifs and 2) to cleave PAR-1. These actions enhance platelet activation by subthreshold doses of ADP leading to $\mathrm{Ca}^{2+}$ mobilization, MAPK-related signaling, and ATP release. Inhibitors to huPK block its plateletpotentiating activity [11-13]. The sum of these investigations suggest how huPK contributes to vascular disease and thrombosis in the intravascular compartment $[1,9]$.

\section{Materials and methods}

\subsection{Collection of human platelets}

Human platelets were obtained from apparently healthy donors at the Charitable Association of Blood Collection - COLSAN São Paulo, SP, Brazil. Blood collection was carried out in accordance with the Declaration of Helsinki and approved by the Institutional Ethics Review Board (CEP1793/11) from the São Paulo Federal University (UNIFESP).

\subsection{Enzymes and reagents}

HuPK was purified as previously described [14] and the specific activity was found to be $80 \mathrm{U} / \mathrm{mg}$. Recombinant Bauhinia bauhinioides kallikrein inhibitor (rBbKI) was obtained as described [15]. ADP, ATP-standard, and luciferin-luciferase were from Chrono-Log (Chester, PA, USA). Human $\alpha$-thrombin (200 NIH units/mg) was from Sigma (St. Louis, MO, USA). The PAR-1 antagonist SCH 79797 was from Cayman Chemical (Ann Arbor, MI, USA); abciximab was from Eli Lilly and Company (Indianapolis, IN, USA), while Fluo-4AM was from Thermo Fisher Scientific Corporation (Waltham, MA, USA). TLCK was purchased from Merck KGaA/Calbiochem (Darmstadt, Germany).

\subsection{Platelet aggregation}

Pooled venous blood obtained from apparently healthy donors was collected into conical plastic tubes containing 3.8\% trisodium citrate $1: 10(\mathrm{v} / \mathrm{v})$. Platelet aggregation was measured using plateletrich plasma (PRP) or washed platelets (WP). Briefly, PRP was obtained by centrifugation at $141 \mathrm{~g}$ for $12 \mathrm{~min}$ and platelet poor plasma (PPP) by centrifugation at $350 \mathrm{~g}$ for $15 \mathrm{~min}$ at room temperature. WP were obtained by centrifugation as previously described [16]. Cells were adjusted to a concentration of $2.5 \times 10^{8}$ platelets/mL with PPP or Hepes Tyrode buffer pH 7.4 (NaCl $134 \mathrm{mM}$, $\mathrm{Na}_{2} \mathrm{HCO}_{3} 12 \mathrm{mM}, \mathrm{KCl} 2.9 \mathrm{mM}, \mathrm{Na}_{2} \mathrm{HPO}_{3} 0.34 \mathrm{mM}, \mathrm{MgCl}_{2} 1.0 \mathrm{mM}$, Hepes $10 \mathrm{mM}, \mathrm{C}_{6} \mathrm{H}_{12} \mathrm{O}_{6} 0.055 \mathrm{mM}$, and $\mathrm{CaCl}_{2} 1 \mathrm{mM}$ ) using a Sysmex KX-21 NTM (Kobe, Hyogo Japan) counter. In a typical experiment, human platelet aggregation was monitored in a two dual-channel modules ChronoLog Model 700 light transmission aggregometer, measuring changes in turbidity with continuous observation over a 6 -min interval after the addition of either ADP $(2.0$ or $10 \mu \mathrm{M})$ or $\alpha$ thrombin (2.0 NIH). PRP and PPP were used to set the light transmission to 0 and $100 \%$. The degree of platelet aggregation was given as percent change in light transmission from the PRP value ( $0 \%$ light transmission) to PPP (100\% light transmission). To test the effects of huPK (40 nM) on aggregation, platelets were previously preincubated with huPK for $3 \mathrm{~min}$ at $37{ }^{\circ} \mathrm{C}$ under stirring, prior to the addition of the subthreshold dose of ADP $(2.0 \mu \mathrm{M})$. In some experiments platelets were separately treated prior to huPK addition with PAR-1 specific antagonist SCH 79797 (140 nM), $\alpha_{\mathrm{IIb}} \beta_{3}$ antagonist abciximab $(1.0 \mu \mathrm{g} / \mathrm{mL})$, huPK recombinant inhibitor rBbKI $(4.0 \mu \mathrm{M})[15]$, TLCK $(340 \mu \mathrm{M})$ or synthetic peptides based on huPK sequence $(5.0 \mu \mathrm{M})$. Subsequently huPK ( $40 \mathrm{nM}$ ) was added to the PRP, pre-incubated for 3 min following addition of the subthreshold dose of ADP. A control measurement was run at the beginning and at the end of each experiment in order to confirm the viability of the platelets.

\subsection{Platelet activation - ATP release}

As described by Gao et al. [17], ADP-induced platelet activation was measured in a lumi-aggregometer (Chronolog). PRP was adjusted to $2.5 \times 10^{8}$ platelets/mL with PPP, supplemented with $1 \mathrm{mM} \mathrm{CaCl}_{2}$ and $0.02 \mathrm{nM}$ luciferin-luciferase reagent, and placed in a siliconized glass cuvette at $37{ }^{\circ} \mathrm{C}$ with constant stirring at $1000 \mathrm{rpm}$. After pre-incubation with huPK $(40 \mathrm{nM})$ for $3 \mathrm{~min}$, platelet activation was initiated by addition of $2.0 \mu \mathrm{M}$ ADP. ATP release was quantified according to the methods recommended by the manufacturer

\subsection{Calcium mobilization}

PRP $\left(2.5 \times 10^{8}\right.$ platelets $\left./ \mathrm{mL}\right)$ was incubated with $4 \mu \mathrm{M}$ Fluo-4$\mathrm{AM}$ at room temperature for $30 \mathrm{~min}$, centrifuged onto coverslips (12 mm diameter) at $141 \mathrm{~g}$ for $5 \mathrm{~min}$ for adhesion of platelets, and then maintained in Hepes-Tyrode buffer. Following centrifugation, intracellular $\mathrm{Ca}^{2+}$ concentrations $\left(\left[\mathrm{Ca}^{2+}\right]_{\mathrm{i}}\right)$ were measured. Platelets stimulated with ADP $(10 \mu \mathrm{M})$ served as control to determine that the platelets were function. Platelets on coverslips also were treated with ADP $(2.0 \mu \mathrm{M})$, huPK $(40 \mathrm{nM})$ or ADP $(2.0 \mu \mathrm{M})$ plus huPK ( $40 \mathrm{nM})$ maintained at $37^{\circ} \mathrm{C}$. Fluo-4-AM was excited with an argon laser $(\lambda E x=488 \mathrm{~nm})$, and the light emission was detected using a Zeiss META detector $(\lambda E m=500-550 \mathrm{~nm})$. Images were collected at approximately $15 \mathrm{~s}$ intervals during $400 \mathrm{~s}$. All images were captured and processed using a LSM 510 META confocal microscope (Zeiss, Germany) with a $63 \times$ objective (Plan-Neofluar, 1.4 numerical aperture) under oil immersion. Fluorescence intensity was normalized to the basal level using Examiner 3.2 (Zeiss, Germany) and Spectralyzer (USA) software.

\subsection{Immunoblot analysis}

Immunoblot analysis was performed with PRP stimulated with ADP $(2.0 \mu \mathrm{M})$ in the absence or presence of huPK $(40 \mathrm{nM})$ for $10 \mathrm{~min}$ at $37{ }^{\circ} \mathrm{C}$. Subsequently, PRP pellets were obtained by centrifugation at $880 \mathrm{~g}$ for $15 \mathrm{~min}$. Platelet detergent lysates were prepared by adding an equal volume of ice-cold $2 \times$ lysis buffer (20 mM Tris, 300 mM NaCl, 2 mM EGTA, 2\% nonidet-P40 pH 7.5) containing $2 \times$ protease and phosphatase inhibitor cocktail (Roche Complete Protease Inhibitor Cocktail), phosphatase inhibitors $1 \mathrm{mM} \mathrm{Na} \mathrm{VO}_{4}$ (sodium orthovanadate), $100 \mathrm{mM} \mathrm{NaF}$ (sodium fluoride) followed by freezing at $-80^{\circ} \mathrm{C}$. The total protein content of PRP was measured with Micro BCA Protein Assay Kit (Pierce). Samples were heated at $95{ }^{\circ} \mathrm{C}$ for $7 \mathrm{~min}$ and then separated according to molecular mass on SDS-PAGE using a $10 \%$ polyacrylamide gel (Merck) in a Mini-PROTEAN II electrophoresis cell (Bio-Rad). The proteins were transferred to a nitrocellulose membrane (GE Healthcare, USA) by $2.4 \mathrm{~h}$ electroblotting at $200-\mathrm{mA}$ constant current in a blotting buffer $(20 \mathrm{mM}$ Tris base, $150 \mathrm{mM}$ glycine, 20\% methanol), using Mini Trans-Blot Electrophoretic Transfer Cell (Bio-Rad). The membranes were quenched for $2 \mathrm{~h}$ with $5 \%$ BSA in TBST buffer $(200 \mathrm{mM}$ Tris/HCl buffer $\mathrm{pH} 8.0$, containing $150 \mathrm{mM} \mathrm{NaCl}$ and $0.05 \%$ Tween 20) and then incubated overnight at $4{ }^{\circ} \mathrm{C}$ with the following primary antibodies: rabbit anti-p38, rabbit anti-phosphoThr ${ }_{180} / \operatorname{Tyr}_{182}$-p38, rabbit anti-phosphoThr $202 / \mathrm{Thr}_{204}$-ERK1/2, rabbit anti-AKT, rabbit anti-phospho AKT-S ${ }^{473}$, and anti- $\beta$-actin (all antibodies from Cell Signaling Technology), diluted in $1 \%$ bovine serum albumin (BSA) in TBST. 
After each step, membranes were sequentially washed three times with TBST. Chemiluminescence signal detection was performed using the gel documentation system ECL (GE Healthcare, United Kingdom) and Super Signal (Pierce, Rockford, IL). The densitometry analysis was performed in the Image software (NIH, USA) using the phospho-proteins/total-proteins ratio and normalized to $\beta$-actin band density.

\subsection{Design of synthetic peptides based on huPK sequence}

The synthetic peptides were designed based on the sequence of

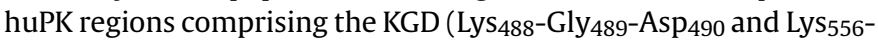
Gly $_{557}-$ Asp $_{558}$ ) and KGE ( Lys $_{509}-$ Gly $_{510}-$ Glu $_{511}$ ) motifs in linear forms. The synthetic peptides based on the primary structure of huPK were designed as Peptide 1 (Ac-C(484)-L-P-S-K-G-D-T-S-T-IY-T-N-C(498)-NH $\mathrm{NH}_{2}$, 1602.8 Da), Peptide 2 (Ac-G(502)-W-G-FS-K-E-K-G-E-I-Q(513)-NH2, MW $1365.62 \mathrm{Da}$ ) and Peptide 3 (AcC(555)-K-G-D-S-G-G-P-L-V-C(565)-NH $\mathrm{N}_{2}$, MW 1035.2).

\subsection{Synthesis of FRET peptide substrates}

The FRET peptide substrates comprising the sequences of the protease activated receptors, PAR-1, -3 , and -4 , were synthesized as described [18]. The molecular mass and purity of the synthesized peptides were assessed by analytical HPLC and by MALDI-TOF using a Microflex-LT (Bruker - Daltonics, Billerica, MA, USA) mass spectrometer. Stock solutions of peptides were prepared in DMF and their concentrations were determined spectrophotometrically using the 2,4-dinitrophenyl group (Dnp) molar extinction coefficient of $17,300 \mathrm{M}^{-1} \mathrm{~cm}^{-1}$ at $365 \mathrm{~nm}$.

\subsection{Hydrolysis of PAR substrates by huPK}

The hydrolysis of FRET peptides by huPK was quantified using a Hitachi F-2500 spectrofluorimeter by measuring the fluorescence at $420 \mathrm{~nm}$ following excitation at $320 \mathrm{~nm}$. HD-Pro-Phe-Arg-MCA, the standard substrate for huPK prepared in DMSO, was assayed as the positive control [18]. The concentration of DMSO or DMF in assay buffers was kept below $1 \%(\mathrm{v} / \mathrm{v})$. The scissile bonds of hydrolyzed peptides were identified by isolation of the fragments using analytical HPLC followed by determination of their molecular mass by LC/MS, using an LCMS-2010 equipped with an ESI-probe (Shimadzu, Japan). Analytical HPLC conditions were: Ultrasphere C18 column $(5 \mu \mathrm{M}, 4.6 \times 250 \mathrm{~mm})$, elution with the solvent system A (water/TFA, 1:1000) and B (ACN/water/TFA 900:100:1), at a flow rate of $1 \mathrm{~mL} / \mathrm{min}$, and a $0-80 \%$ gradient for $20 \mathrm{~min}$, monitored by absorbance at $220 \mathrm{~nm}$. The kinetic parameters $\mathrm{K}_{\mathrm{M}}$ and $\mathrm{k}_{\text {cat }}$ were calculated by nonlinear regression using the GraFit software. HuPK concentration was adjusted to hydrolyze less than $5 \%$ of the substrate over the course of the assay. The reaction rate was converted into micromoles of substrate hydrolyzed per minute based on a calibration curve obtained from the complete hydrolysis of each
(A)

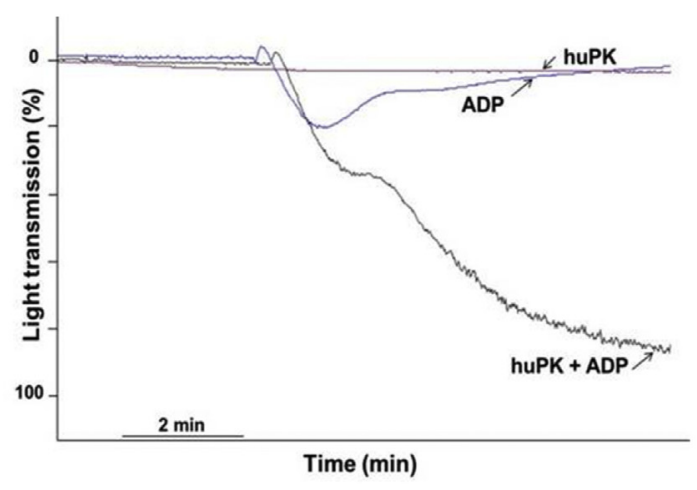

(C)

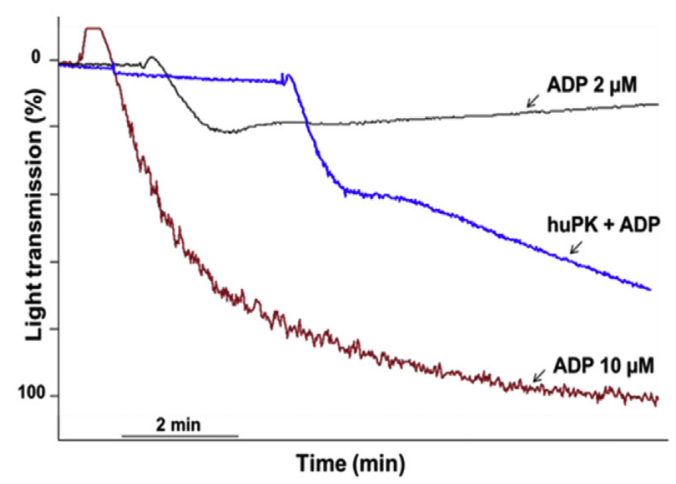

(B)

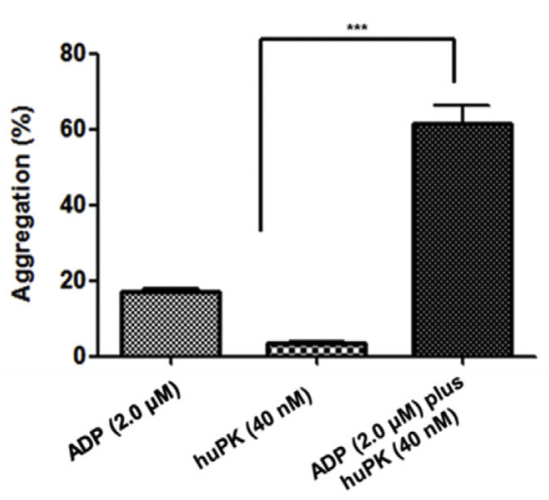

(D)

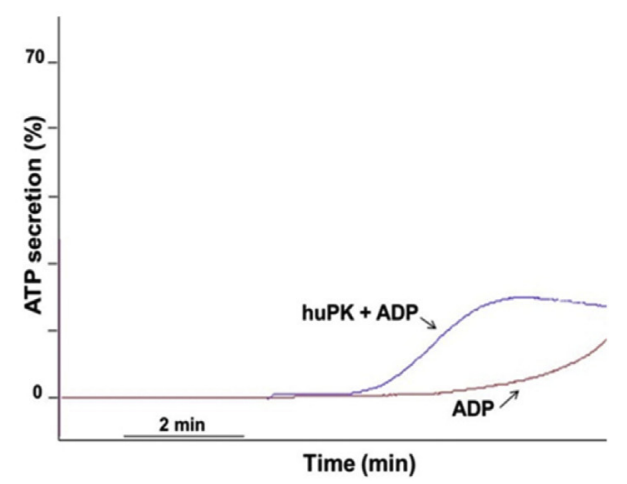

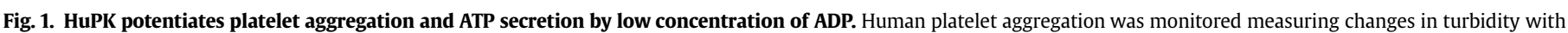

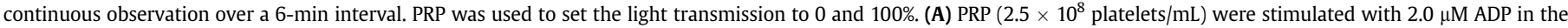

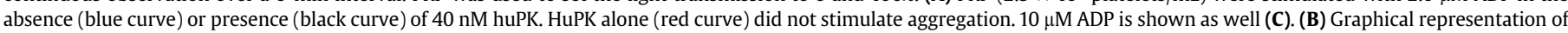

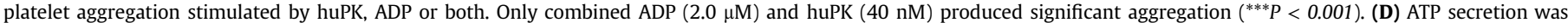

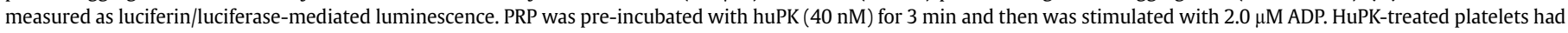
enhanced ATP release over ADP alone. HuPK alone did not stimulate granule secretion (data not show). The figure is a representative of 3 studies. 
(A)
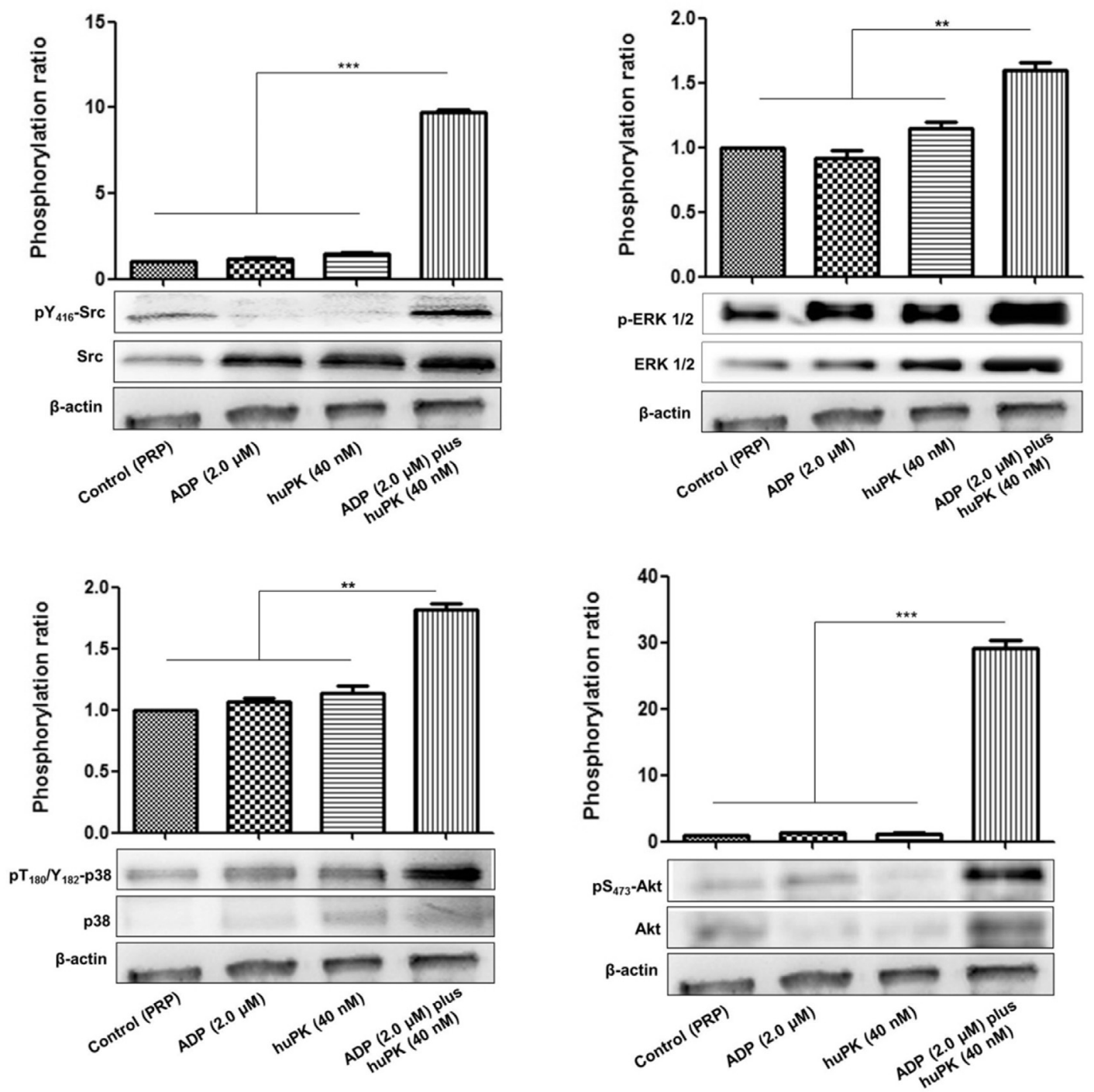

(B)

(C)
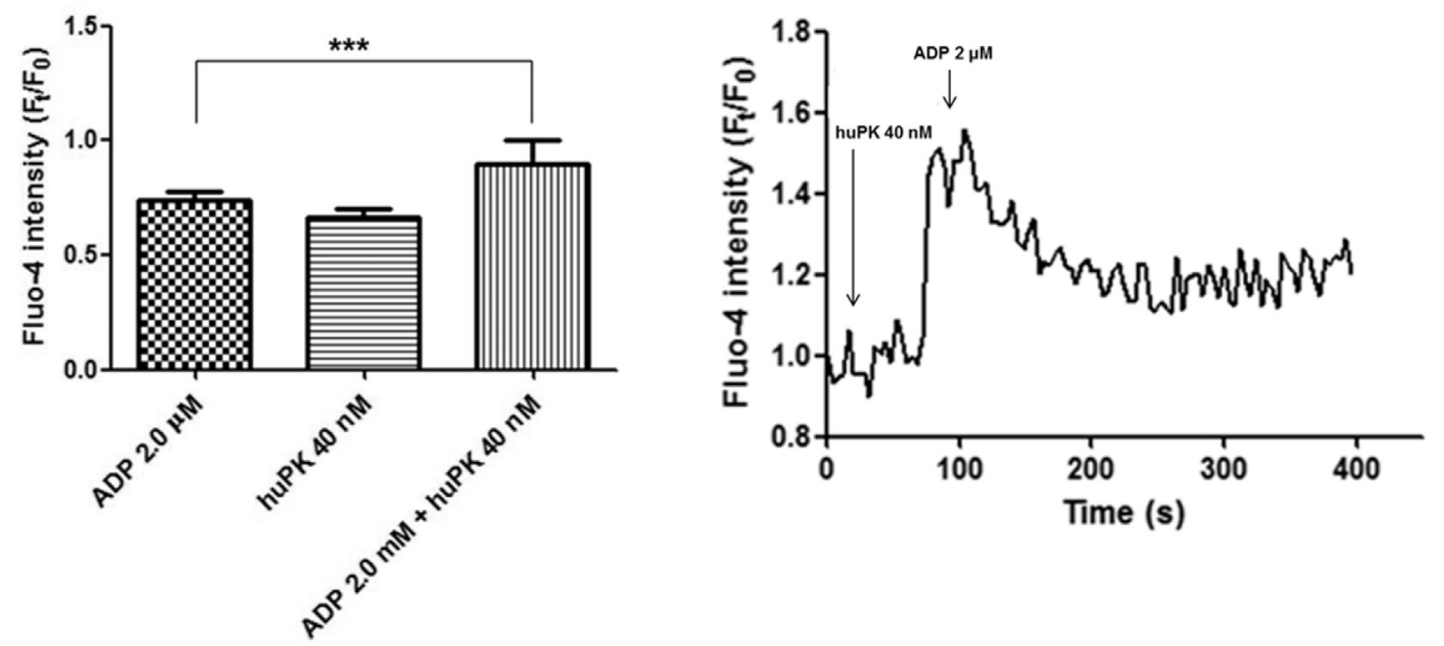

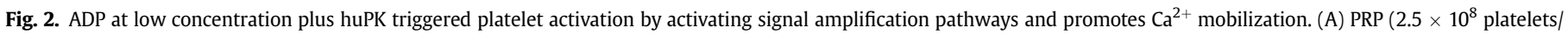

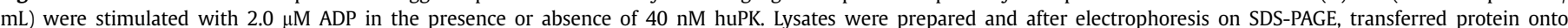

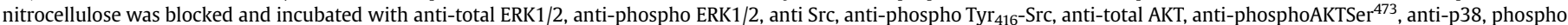


peptide. The data were fitted with respective standard errors to the Michaelis-Menten equation using GraFit software (Erithacus Software Limited). In all assays, data were collected at least in duplicate and the error values were less than $10 \%$ for each of the obtained kinetic parameters.

\subsection{Statistical analysis}

The results from the in vitro studies are presented as the means of three independent experiments. The statistical analysis was performed using GraphPad Prism5. Briefly, the Student's t-test was used to compare means between two independent groups, whereas one-way ANOVA followed by the Tukey's post-test, was used to compare the means between two or more independent groups. Two-way ANOVA was used to compare group means influenced by two independent factors. Data represent means $\pm S D$ of three independent experiments. Each experimental condition was performed in triplicate. The level of $\mathrm{p} \leq 0.05$ was accepted as significant.

\section{Results}

\subsection{HuPK potentiates platelet aggregation and granule release at low concentration of $A D P$}

ADP evokes either reversible or irreversible platelet aggregation depending on its concentration and the sensitivity of the platelet sample to it. We previously demonstrated [10], huPK potentiates ADP-induced platelet aggregation, converting subthreshold reversible aggregation to irreversible aggregation. Two $\mu \mathrm{M}$ or less ADP-induced platelet aggregation was reversible with maximal chart deflection at $29 \pm 1.8 \%$ (Fig. $1 \mathrm{~A}$ ). Ten $\mu \mathrm{M}$ ADP induced irreversible platelet aggregation (Fig. 1C). Forty nM huPK failed to induce any measurable aggregation (Fig. 1A). When human PRP was pre-incubated for $3 \mathrm{~min}$ at $37^{\circ} \mathrm{C}$ with $40 \mathrm{nM}$ huPK, $2.0 \mu \mathrm{M}$ ADP induced both primary and secondary wave aggregation with a maximal aggregation of $80 \pm 1.0 \%$ (Fig. $1 \mathrm{~A}$ ). In three independent experiments, $2.0 \mu \mathrm{M}$ ADP incubated with PRP pre-treated with $40 \mathrm{nM}$ huPK significantly $(P<0.001)$ increased the \% maximal aggregation over ADP or huPK alone (Fig. 1B). Additional investigations showed that the primary and secondary wave $2.0 \mu \mathrm{M}$ ADP-induced platelet aggregation induced by huPK-primed platelets also were associated with ATP secretion. ADP-treated platelets had enhanced ATP release in the presence of previously huPKtreated platelet (Fig. 1D). Although huPK alone did not stimulate either aggregation or granule secretion, it potentiated the effect of $2 \mu \mathrm{M}$ ADP in both reactions.

\subsection{HuPK-primed platelets allow for $2.0 \mu M$ ADP-induced pERK1/2 and p38 mitogen-activated protein kinase (MAPK) phosphorylation in human platelets}

ADP-induced platelet activation leads to ERK1/2 (extracellular signal-regulated kinase $1 / 2$ ) and Akt $S^{473}$ (serine/threonine kinase) phosphorylation through Src family tyrosine kinases, such as JNK1 (c-Jun N-terminal kinase-1), and consequently p38 MAPK activation. These events depend on the signaling from the ADP receptors
(P2Y1 and the P2Y12 receptors), resulting in the cascade of platelet activation events that leads in the formation of a stable thrombus. The MAPK pathway also is triggered through PARs activation and integrin $\alpha \operatorname{Ilb} \beta 3$ outside-in signaling and dependent on the release of the contents of the platelet granules $[19,20]$. The biochemical mechanisms after low concentration ADP stimulation downstream from huPK primed platelets was investigated. Immunoblot studies showed that when $40 \mathrm{nM}$ huPK primed platelets, $2.0 \mu \mathrm{M}$ ADP stimulation increased pSrc $\mathrm{Y}^{416}$, pERK1/2, p38 MAPK, and pAkt $S^{473}$ (Fig. 2A). HuPK (40 nM) or ADP $(2 \mu \mathrm{M})$ alone, however, did not induce phosphorylation of any of hese platelet proteins to the same extent.

\subsection{ADP plus huPK promotes $\mathrm{Ca}^{2+}$ mobilization}

To assess the ability of ADP plus huPK to induce Ca2+ release during platelet aggregation, PRP was loaded with the Fluo-4AM. PRP treated with ADP $(10 \mu \mathrm{M})$ induced irreversible platelet aggregation (Fig. 1C) and triggered a robust transient increase of cytoplasmic $\mathrm{Ca} 2+$ concentration (data not shown) as described by Koessler et al. [21]. On the other hand, ADP $(2.0 \mu \mathrm{M})$ alone did not significantly induce cytoplasmic Ca2+ mobilization (Fig. 2B). Likewise, huPK (40 nM) alone did not significantly increase Ca2+ mobilization (Fig. 2B). When examined graphically, huPK (40 nM) resulted in an increase of the average amplitude of $\mathrm{Ft} / \mathrm{FO}$ $(0.7 \pm 0.04)$ showing slightly sustained level after $\sim 400 \mathrm{~s}$ (Fig. 2C). However, when platelets were stimulated further by the addition of $\operatorname{ADP}(2.0 \mu \mathrm{M})$ in the same reaction, there was a robust transient $\mathrm{Ca} 2+$ release from granules, with significant average amplitude of Ft/F0 in $0.9 \pm 0.1$ (Fig. 2B and C).

\subsection{Is the effect of huPK dependent on its proteolytic activity via protease-activated receptors?}

Since huPK is a trypsin like protease and has been shown to cleave PAR-1, we asked if PAR-1 contributed to the process [7]. Initial studies showed that huPK's contribution to ADP-induced platelet activation was a proteolytic process since the specific reversible plasma kallikrein inhibitor, recombinant BbKI $(4.0 \mu \mathrm{M})$, and the general serine protease inhibitor TLCK $(340 \mu \mathrm{M})$ were able to block the huPK potentiation of $2.0 \mu \mathrm{M}$ ADP-induced platelet aggregation (Fig. 3A). Additionally, the specific PAR-1 receptor antagonist SCH $79797(140 \mathrm{nM})$ also inhibited huPK primed $2.0 \mu \mathrm{M}$ ADP-induced platelet aggregation (Fig. 3B). Synthetic FRET substrate peptides derived from human PAR-1 and PAR-3, but not PAR4 , encompassing their $\alpha$-thrombin cleavage sites were hydrolyzed by plasma kallikrein (Table 1 ). These combined studies suggested that PAR-1 is a candidate protease-activated substrate for huPK potentially priming platelets for ADP-induced activation.

\subsection{HuPK motifs for recognition and binding to platelets}

Additional investigations elucidated how huPK binds to platelets to activate PAR-1. HuPK does not have exosite regions that bind substrates such as $\alpha$-thrombin [2,22]. However, huPK contains two KGD (Lys $488-$ Gly $_{489}-$ Asp $_{490}$ and Lys $_{556}-$ Gly $_{557}-$ Asp $_{558}$ ) motifs and one KGE (Lys $509-$ Gly $_{510}-$ Glu $\left._{511}\right)$ motif in its light chain (Fig. 4A). The

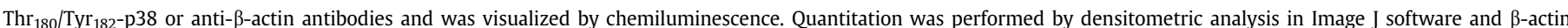

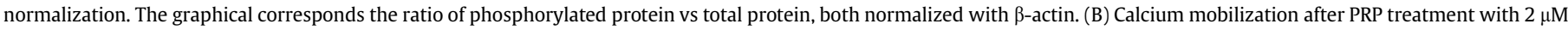

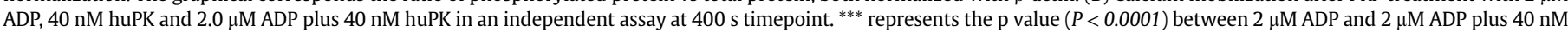

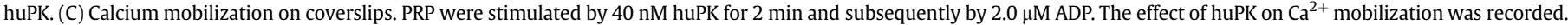

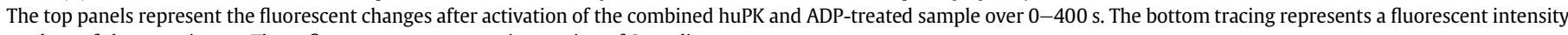
readout of the experiment. These figures are representative tracing of 3 studies. 
(A)
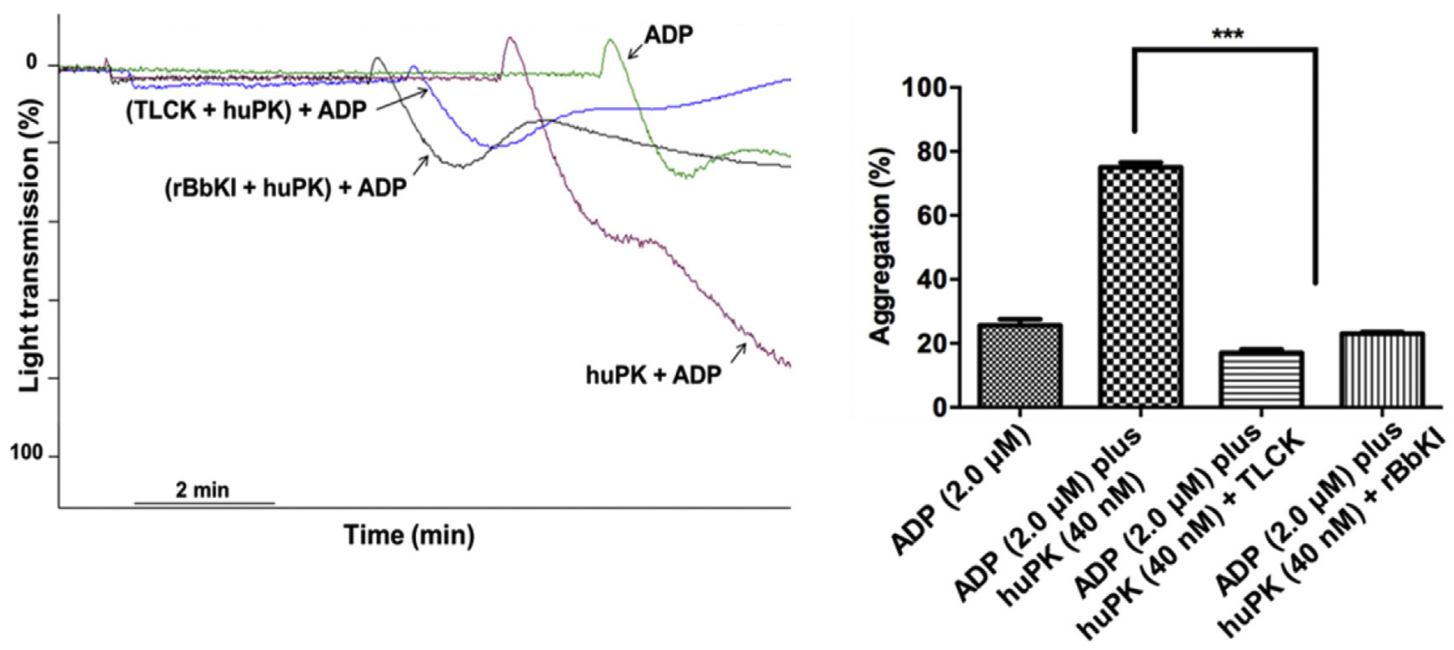

(B)
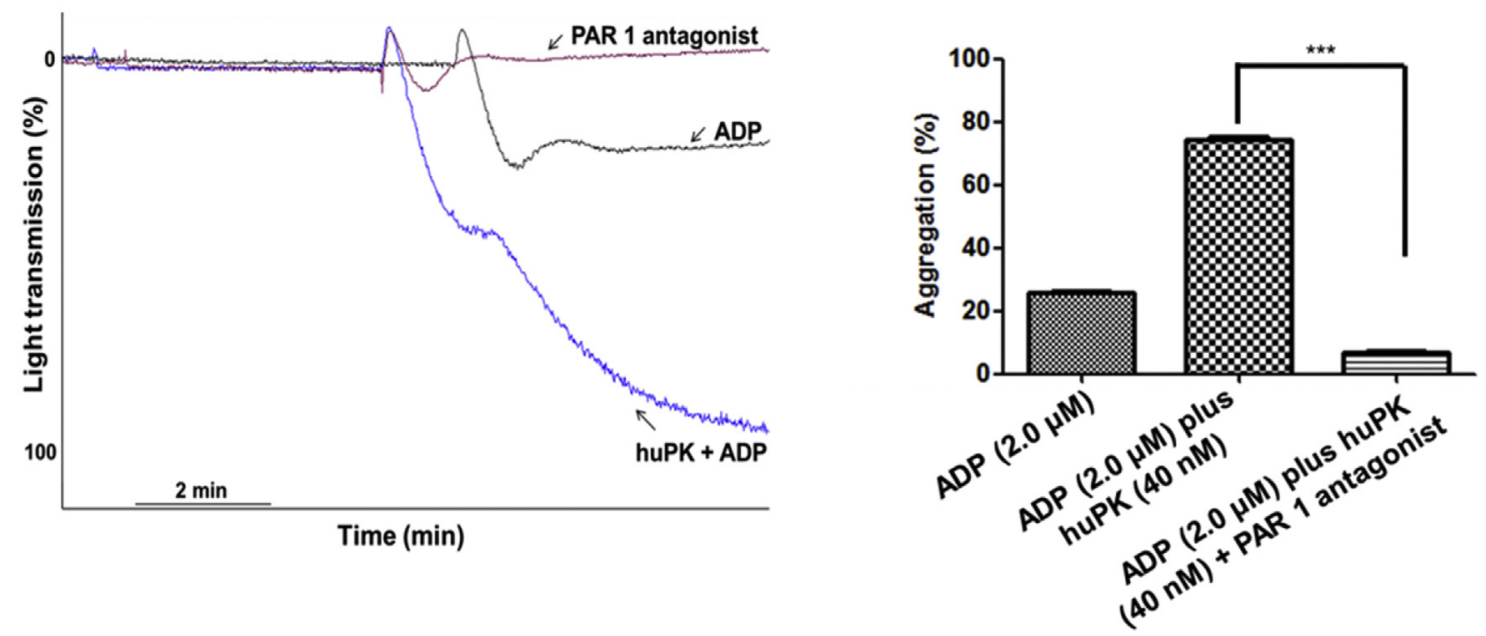

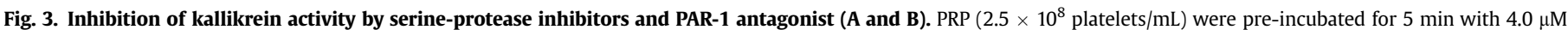

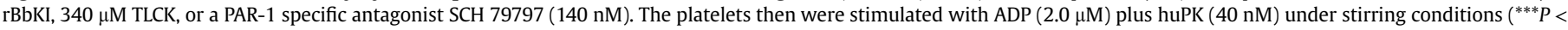
$0.0001)$. These figures are representative tracing of 3 studies.

Table 1

Kinetic parameters for the Hydrolysis of the FRET peptides derived from sequence that span the activation cleavage sites of protease-activated receptors (PAR) 1, 3 and 4 by huPK.

\begin{tabular}{lll}
\hline Peptide & Abz-peptidyl-EDDnp & $\mathrm{k}_{\text {cat }} / \mathrm{K}_{\mathrm{M}}\left(10^{6} \mathrm{M}^{-1} \mathrm{~s}^{-1}\right)$ \\
\hline PAR 1 & Abz-TLDPR $\downarrow$ SFLLQ-EDDnp & a \\
PAR 3 & Abz-TLPIKTFR $\downarrow$ GQ-EDDnp & 2.8 \\
PAR 4 & Abz-LPAPRGYPGQ-EDDnp & No Hydrolysis \\
\hline
\end{tabular}

The following conditions for substrate hydrolysis were used: $50 \mathrm{mM}$ Tris- $\mathrm{HCl}, \mathrm{pH}$ 8.0. All the reactions were at $37^{\circ} \mathrm{C}$. The arrow $(\downarrow)$ indicates the cleaved peptide bonds determined by fragment identification by LC/MS.

a Both cleavage sites were equally hydrolyzed at the PAR-1 peptide.

KGD/RGD sequence is a specific sequence motif for adhesive proteins $[2,22,23]$ and interacts with $\alpha \operatorname{IIb} \beta 3$ integrin $[2,22,24]$. The binding of the KGD sequence induces cell signaling leading to phosphorylation of the Y759 residue located in the $\beta 3$ integrin cytoplasmic domain of integrin [25]. The three-dimensional structure of HuPK indicates that the Lys ${ }_{556}-$ Gly $_{557}-$ Asp $_{558}$ (KGE) sequence is close to the active site $\operatorname{Ser}_{559}$ (Fig. 4B) whereas the KGD sequences are more distant, but in a location on the surface of the molecule (Fig. 4). Thus, we postulated that these sequences may bind integrin $\alpha_{\mathrm{IIb}} \beta_{3}$. This premise was confirmed in PRP by the lack of the huPK potentiating effect of platelet aggregation in the presence of the receptor antagonist abciximab $(1.0 \mu \mathrm{g} / \mathrm{mL})$ (Fig. 5A) and by using synthetic peptides derived from plasma kallikrein. When human platelets were pre-incubated with $5 \mu \mathrm{M}$ synthetic huPK peptides (see Methods) that contained the KGD and KGE sequences (peptides 1, 2 and 3), each peptide blocked the effect of $40 \mathrm{nM}$ huPK on ADP-induced platelet aggregation (Fig. 5B), disrupting huPK- $\alpha$ IIb $\beta 3$ binding and consequently its proteolytic activity on the PAR receptors. Studies with washed platelets (WP) confirmed that ADP $(2 \mu \mathrm{M})$ alone was not a sufficient agonist [21] and neither huPK alone nor when combined with $2 \mu \mathrm{M}$ ADP induced platelet aggregation as occurs in PRP (Fig. 6A) as well the peptides comprising the KGD and KGE sequence (Fig. 6B). These results suggest that stimulation is required for the $\alpha$ IIb $\beta 3$ integrin to be activated and to expose the recognition site to the KGD and KGE sequences used by kallikrein for its anchorage. Thrombin, the PAR-1 agonist, added in the WP pre-incubated with ADP plus huPK, 
(A)

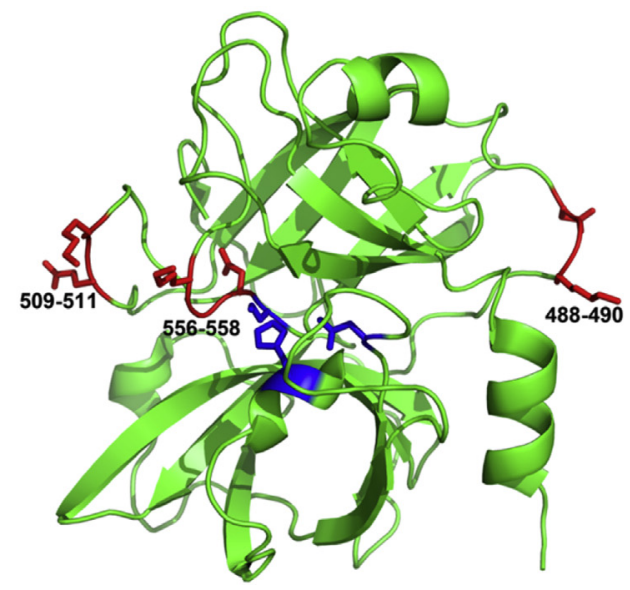

(B)

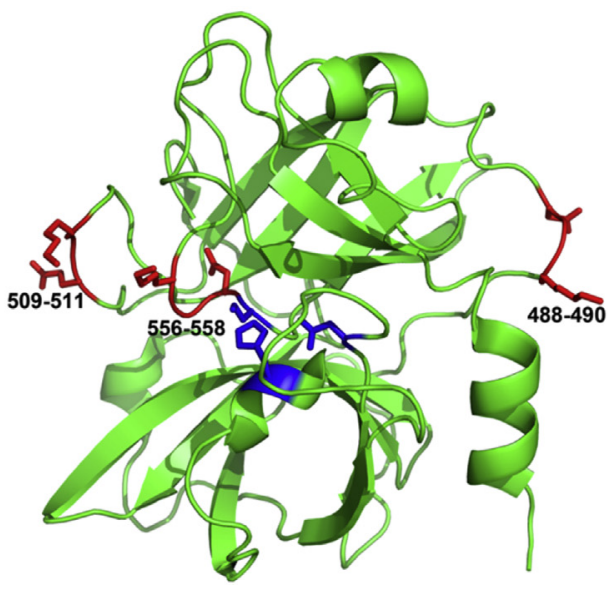

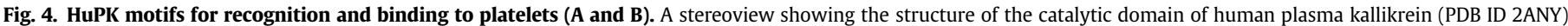

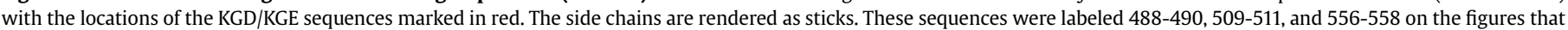

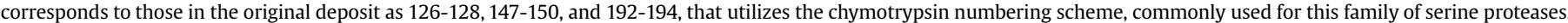
$\mathrm{S}_{559}, \mathrm{D}_{464}$ and $\mathrm{H}_{434}$ of huPK active site are in blue.

(A)
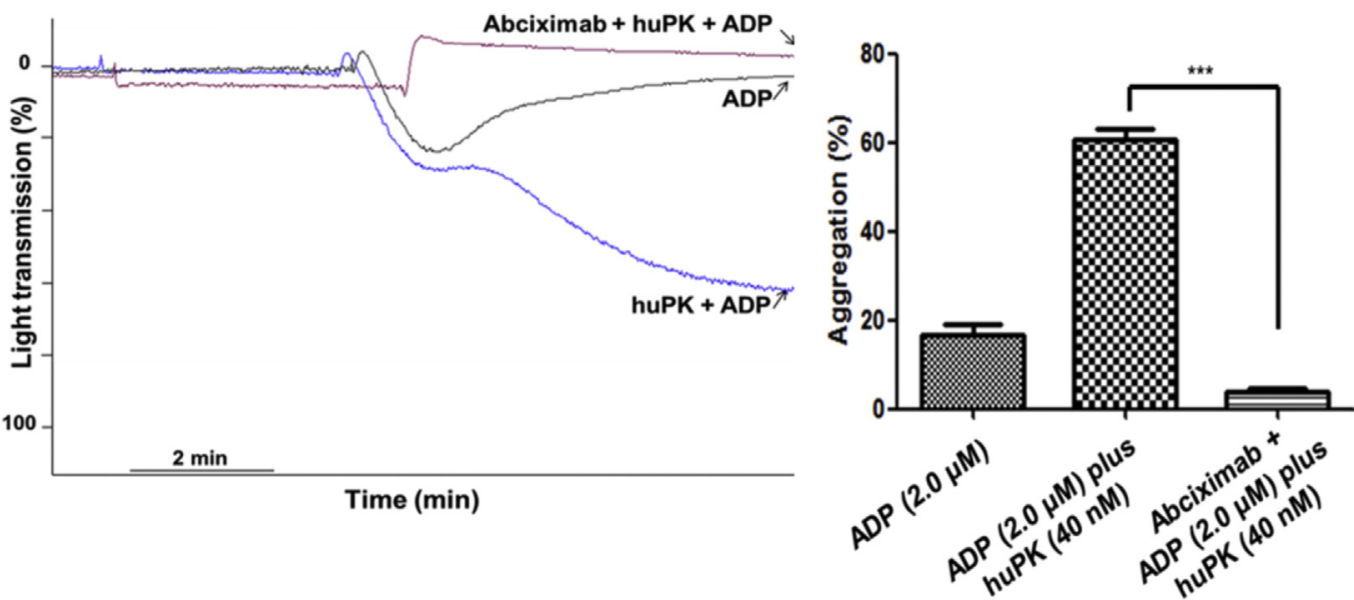

(B)
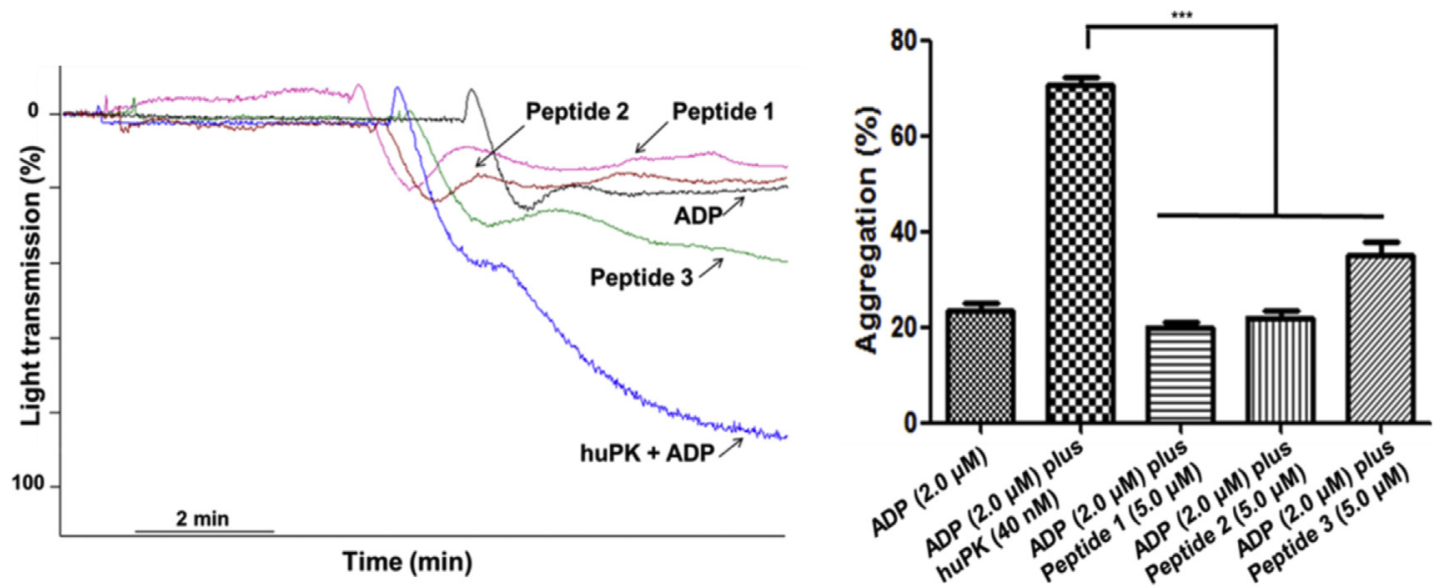

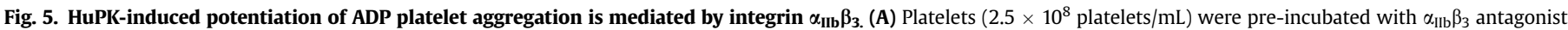

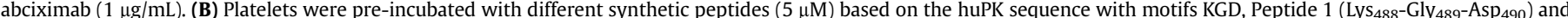

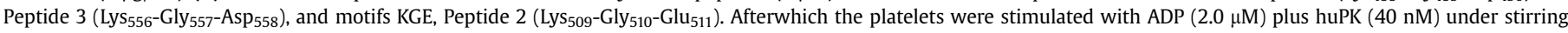
conditions $\left({ }^{* * *} P<0.0001\right)$. These figures are representative tracing of 3 studies. 
(A)

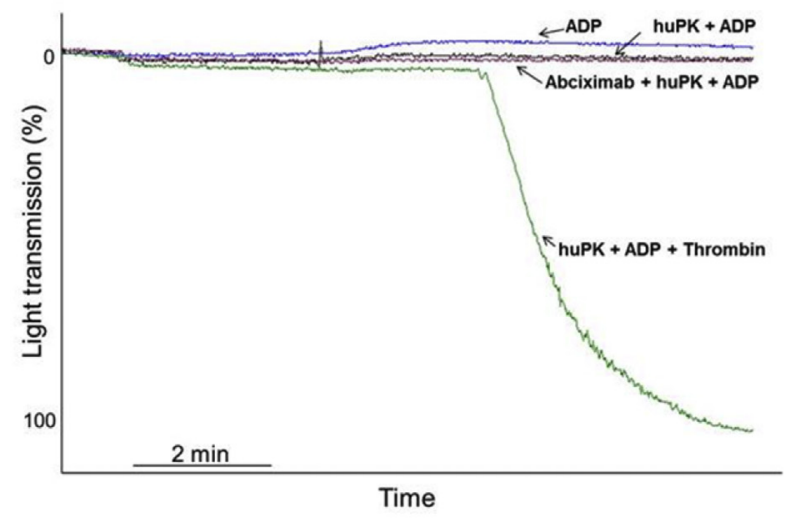

(B)

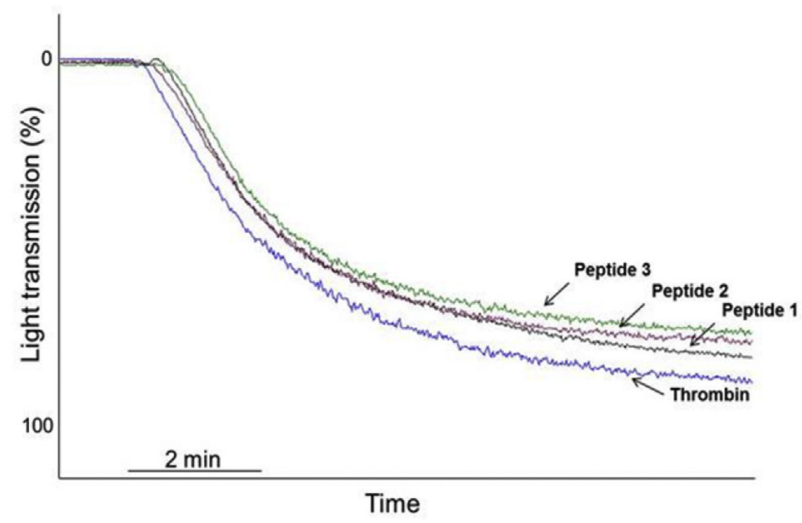

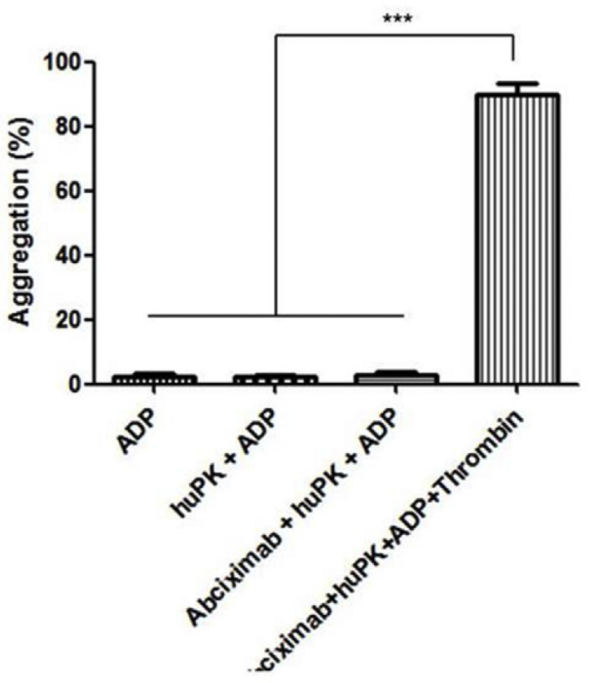

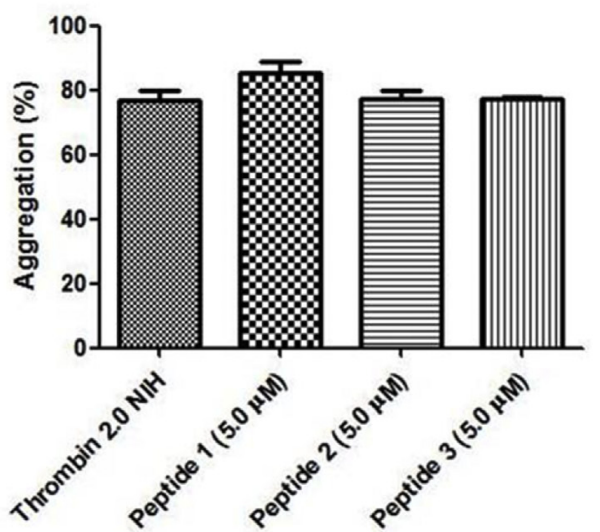

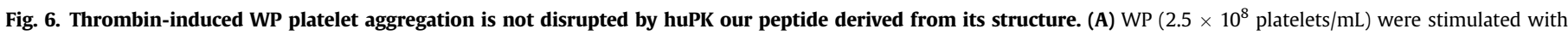

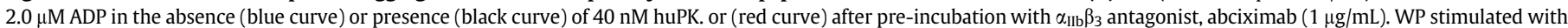

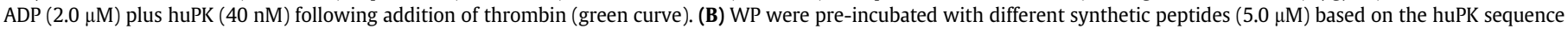
with motifs KGD, Peptide 1, Peptide 3, and motifs KGE, Peptide 2 following addition of thrombin. ( ${ }^{* * *} P<0.0001$ ). These figures are representative tracing of 3 studies.

peptides or abciximab, was able to induce platelets aggregation, indicating that the crosstalk between $\alpha$ IIb $\beta 3$ and PAR- 1 signaling on the intracellular cascade level is not crucial to this potent platelet agonist unlike in the case of huPK.

\section{Discussion}

In 1965, Werle and Schievelbein [10] reported that high concentrations of huPK were not able to initiate platelet aggregation and did not influence the aggregation elicited by physiological amount of ADP. In agreement with Cassaro et al. [11] we observe that huPK in PRP is able to reduce the threshold of ADP-induced platelet activation. It is quite remarkable that the addition of active enzyme to PRP is able to alter platelets such that low concentrations of ADP are able to induced irreversible platelet aggregation.

Stimulation of platelets with a variety of physiological stimuli, including ADP, initiates the primary reversible phase of aggregation [26]. It is well established that ADP can evoke either reversible or irreversible platelet aggregation, depending on its concentration and the responsiveness of the platelet samples. Adequate platelet activation leads to secretion of their granule contents in an aggregation-dependent manner with recruitment of additional platelets, giving rise to the irreversible phase of aggregation $[19,27,28]$. Sub-physiological doses of ADP (e.g. $2.0 \mu \mathrm{M}$ ) are not sufficient to initiate the second wave of platelet aggregation [20,28-32]. Our findings clarify our previous study [10] which show that huPK potentiates ADP-induced platelet aggregation at sub-physiological doses. HuPK appears to do this by possibly 1) binding to $\alpha_{\mathrm{IIb}} \beta_{3}$ integrin and 2) cleaving PAR-1. This "priming" appears to be necessary for lowering the ADP-induced threshold of platelet activation. How cleavage of PAR1 by huPK primes the platelet to respond to lower doses of ADP is not completely known. HuPK itself did not induce aggregation nor granule secretions. More investigations are needed to determine if huPK primes platelets for subthreshold activation by $\alpha$-thrombin, collagen and related GPVI activators or is this just unique to ADP.

Our data show that synergic effect of huPK and subdoses of ADP promote MAPK ERK1/2, pSrc and pAKT-S $S^{473}$ phosphorilation. Some authors [33-35] have shown that several platelet surface receptors 
activate MAPK signaling. PAR-4 activation induces full platelet spreading on a fibrinogen matrix with involvement of ERK2, p38 phosphorylation, and $\mathrm{Ca}^{2+}$ mobilizaton. Also, Marshall et al. [36] demonstrated that the Src kinase-dependent signaling pathway, and not MAPK, is involved in integrin $\alpha_{\mathrm{II}} \beta_{3}$ activation induced by von Willebrand factor. In contrast, Li et al. [37] showed that activation of $\alpha_{\text {IIb }} \beta_{3}$ by von Willebrand factor binding to its receptor, glycoprotein Ib-IX-V, is dependent on protein kinase G and MAPK. The association of $40 \mathrm{nM}$ huPK and $2 \mu \mathrm{M}$ ADP may increases the receptor-mediated response sufficiently such that threshold protein phosphorylation and calcium mobilization occur.

Our studies also show that the proteolytic activity of huPK is necessary for its ability to lower the threshold of ADP-induced platelet activation. The fact that a specific PAR-1 antagonist blocks this ability of huPK strongly suggests that PAR-1 participates in the reaction. This information plus the known information that PAR-1 is a substrate of huPK indicate PAR-1's role in this proecess [7]. Proteolysis of PAR-1 may allow for a lower concentration of ADP to activate the $\mathrm{P} 2 \mathrm{Y}^{12}$ receptor for its platelet activation.

It is not completely known how huPK interacts with the surface of platelets to trigger platelet activation. Our data propose that the event may be initiated by huPK binding through its KGD/KGE motifs since peptides to this region block platelet aggregation. It is known that prekallikrein binds to cells through both high molecular weight kininogen and non-kininogen interaction motifs [39]. Integrins may represent a candidate non-kininogen binding site for prekallikrein.

In sum, these findings unveil a novel and not previously appreciated aspect of a non-canonical huPK activity in platelet activation. Our results should prompt further investigations into the effects of this pathway leading to platelet activation and subsequent clot formation.

\section{Conflict of interests}

The authors state that they have no conflict of interest.

\section{Authors' contributions}

T.F. Ottaiano and S.S. Andrade contributed to assay design and performance, interpretation of the data, statistical analysis, and wrote the text; C. de Oliveira, M.V. Buri and M.C.C. Silva performed the in vitro assays; M.L.V. Oliva directed the coordination of the study, contributed to results interpretation and wrote the text; M.A. Juliano, M.J.B.C. Girão, F.H.A. Maffei, M.U. Sampaio, A. H. Schmaier and $A$. Wlodawer contributed to structural analysis and manuscript preparation. All authors read and approved the final version of this manuscript.

\section{Acknowledgments}

We thank to Fundação de Amparo à Pesquisa do Estado de São Paulo Proc. 2009/53766-5 and 2012/19780-3, Coordenação de Aperfeiçoamento de Pessoal de Nível Superior (CAPES/Drug Discovery AUXPE 140/2015, Proc. 23038.007776/2014-32), Conselho Nacional de Desenvolvimento Científico e Tecnológico Proc. 142009/2012-1, Associação Beneficente de Coleta de Sangue do Estado de São Paulo (COLSAN) for their financial support. This work was supported in part by the Intramural Research Program of the $\mathrm{NIH}$, National Cancer Institute, Center for Cancer Research.

\section{References}

[1] E.P. Feener, Q. Zhou, W. Fickweiler, Role of plasma kallikrein in diabetes and metabolism, Thromb. Haemost. 110 (2013) 434-441.
[2] I.M. Sainz, R.A. Pixley, R.W. Colman, Fifty years of research on the plasma kallikrein-kinin system: from protein structure and function to cell biology and in-vivo pathophysiology, Thromb. Haemost. 98 (2007) 77-83.

[3] E. Fink, K.D. Bhoola, C. Snyman, P. Neth, C.D. Figueroa, Cellular expression of plasma prekallikrein in human tissues, Biol. Chem. 388 (2007) 957-963.

[4] P. Neth, M. Arnhold, H. Nitschko, E. Fink, The mRNAs of prekallikrein, factors $\mathrm{XI}$ and XII, and kininogen, components of the contact phase cascade are differentially expressed in multiple non-hepatic human tissues, Thromb. Haemost. 85 (2001) 1043-1047.

[5] T. Renne, J. Dedio, J.C. Meijers, D. Chung, W. Muller-Esterl, Mapping of the discontinuous H-kininogen binding site of plasma prekallikrein. Evidence for a critical role of apple domain-2, J. Biol. Chem. 274 (1999) 25777-25784.

[6] F. Marceau, D. Regoli, Bradykinin receptor ligands: therapeutic perspectives, Nat. Rev. Drug Discov. 3 (2004) 845-852.

[7] R.T. Abdallah, J.S. Keum, H.M. El-Shewy, M.H. Lee, B. Wang, M. Gooz D.K. Luttrell, L.M. Luttrell, A.A. Jaffa, Plasma kallikrein promotes epidermal growth factor receptor transactivation and signaling in vascular smooth muscle through direct activation of protease-activated receptors, J. Biol. Chem. 285 (2010) 35206-35215.

[8] J. Bjorkqvist, A. Jamsa, T. Renne, Plasma kallikrein: the bradykinin-producing enzyme, Thromb. Haemost. 110 (2013) 399-407.

[9] A.A. Jaffa, R. Durazo-Arvizu, D. Zheng, D.T. Lackland, S. Srikanth, W.T. Garvey A.H. Schmaier, D.E.S. Group, Plasma prekallikrein: a risk marker for hypertension and nephropathy in type 1 diabetes, Diabetes 52 (2003) 1215-1221.

[10] C.M. Cassaro, M.U. Sampaio, N.Y. Maeda, D.F. Chamone, C.A. Sampaio, Human plasma kallikrein: effect on the induced platelet aggregation, Thromb. Res. 48 (1987) 81-87.

[11] M.L. Oliva, S.A. Andrade, M.A. Juliano, R.C. Sallai, R.J. Torquato, M.U. Sampaio V.J. Pott, C.A. Sampaio, Kinetic characterization of factor Xa binding using a quenched fluorescent substrate based on the reactive site of factor Xa inhibitor from Bauhinia ungulata seeds, Curr. Med. Chem. 10 (2003) 1085-1093.

[12] M.L. Oliva, C.R. Mendes, M.A. Juliano, J.R. Chagas, J.C. Rosa, L.J. Greene, M.U. Sampaio, C.A. Sampaio, Characterization of a tissue kallikrein inhibitor isolated from Bauhinia bauhinioides seeds: inhibition of the hydrolysis of kininogen related substrates, Immunopharmacology 45 (1999) 163-169.

[13] M.L. Oliva, M.U. Sampaio, Action of plant proteinase inhibitors on enzymes of physiopathological importance, An Acad Bras Cienc 81 (2009) 615-621.

[14] M.L. Oliva, M.U. Sampaio, C.A. Sampaio, Isolation and characterization of plant inhibitors directed against plasma kallikrein and factor XII, Adv. Exp. Med Biol. 247A (1989) 467-471.

[15] A.P. Araujo, D. Hansen, D.F. Vieira, C. Oliveira, L.A. Santana, L.M. Beltramini C.A. Sampaio, M.U. Sampaio, M.L. Oliva, Kunitz-type Bauhinia bauhinioides inhibitors devoid of disulfide bridges: isolation of the cDNAs, heterologous expression and structural studies, Biol. Chem. 386 (2005) 561-568.

[16] S.S. Andrade, M.C. Silva, I.E. Gouvea, M.Y. Kondo, M.A. Juliano, M.U. Sampaio, M.L. Oliva, Baupain, a plant cysteine proteinase that hinders thrombininduced human platelet aggregation, Protein Pept. Lett. 19 (2012) 474-477.

[17] C. Gao, B. Boylan, J. Fang, D.A. Wilcox, D.K. Newman, P.J. Newman, Heparin promotes platelet responsiveness by potentiating alphallbbeta3-mediated outside-in signaling, Blood 117 (2011) 4946-4952.

[18] P.F. Angelo, A.R. Lima, F.M. Alves, S.I. Blaber, I.A. Scarisbrick, M. Blaber, L. Juliano, M.A. Juliano, Substrate specificity of human kallikrein 6: salt and glycosaminoglycan activation effects, J. Biol. Chem. 281 (2006) 3116-3126.

[19] A. Garcia, H. Shankar, S. Murugappan, S. Kim, S.P. Kunapuli, Regulation and functional consequences of ADP receptor-mediated ERK2 activation in platelets, Biochem. J. 404 (2007) 299-308.

[20] A. Mazharian, S. Roger, E. Berrou, F. Adam, A. Kauskot, P. Nurden, M. JandrotPerrus, M. Bryckaert, Protease-activating receptor-4 induces full platelet spreading on a fibrinogen matrix: involvement of ERK2 and p38 and Ca2+ mobilization, J. Biol. Chem. 282 (2007) 5478-5487.

[21] J. Koessler, S. Hermann, K. Weber, A. Koessler, S. Kuhn, M. Boeck, A. Kobsar, Role of Purinergic receptor expression and function for reduced responsiveness to adenosine diphosphate in washed human platelets, PloS One 11 (2016) e0147370.

[22] E. De Candia, Mechanisms of platelet activation by thrombin: a short history, Thromb. Res. 129 (2012) 250-256.

[23] S.M. Strukova, Role of platelets and serine proteinases in coupling of blood coagulation and inflammation, Biochem. Biokhimiia 69 (2004) 1067-1081.

[24] K.S. Prasad, P. Andre, M. He, M. Bao, J. Manganello, D.R. Phillips, Soluble CD40 ligand induces beta3 integrin tyrosine phosphorylation and triggers platelet activation by outside-in signaling, Proc. Natl. Acad. Sci. U. S. A. 100 (2003) $12367-12371$.

[25] D.C. Snell, V. Schulte, G.E. Jarvis, K. Arase, D. Sakurai, T. Saito, S.P. Watson, B. Nieswandt, Differential effects of reduced glycoprotein VI levels on activation of murine platelets by glycoprotein VI ligands, Biochem. J. 368 (2002) 293-300.

[26] S. Murugappa, S.P. Kunapuli, The role of ADP receptors in platelet function, Front. Biosci. 11 (2006) 1977-1986.

[27] L.J. Gay, B. Felding-Habermann, Contribution of platelets to tumour metastasis, Nat. Rev. Cancer 11 (2011) 123-134.

[28] L. Leung, R. Nachman, Molecular mechanisms of platelet aggregation, Annu. Rev. Med. 37 (1986) 179-186.

[29] M.H. Cobb, T.G. Boulton, D.J. Robbins, Extracellular signal-regulated kinases: ERKs in progress, Cell Regul. 2 (1991) 965-978. 
[30] L. Jiang, C. Xu, S. Yu, P. Liu, D. Luo, Q. Zhou, C. Gao, H. Hu, A critical role of thrombin/PAR-1 in ADP-induced platelet secretion and the second wave of aggregation, J. Thromb. Haemost. 11 (2013) 930-940.

[31] F. Maione, C. Cicala, E. Liverani, N. Mascolo, M. Perretti, F. D'Acquisto, IL-17A increases ADP-induced platelet aggregation, Biochem. Biophys. Res. Commun. 408 (2011) 658-662.

[32] L. Zhou, A.H. Schmaier, Platelet aggregation testing in platelet-rich plasma: description of procedures with the aim to develop standards in the field, Am. J. Clin. Pathology 123 (2005) 172-183.

[33] F. Nadal, S. Levy-Toledano, F. Grelac, J.P. Caen, J.P. Rosa, M. Bryckaert, Negative regulation of mitogen-activated protein kinase activation by integrin alphaIIbbeta3 in platelets, J. Biol. Chem. 272 (1997) 22381-22384.
[34] Y.A. Senis, A. Mazharian, J. Mori, Src family kinases: at the forefront of platelet activation, Blood 124 (2014) 2013-2024.

[35] S.P. Watson, J.M. Auger, O.J. McCarty, A.C. Pearce, GPVI and integrin alphaIIb beta3 signaling in platelets, J. Thromb. Haemost. 3 (2005) 1752-1762.

[36] S.J. Marshall, Y.A. Senis, J.M. Auger, R. Feil, F. Hofmann, G. Salmon, J.T. Peterson, F. Burslem, S.P. Watson, GPIb-dependent platelet activation is dependent on Src kinases but not MAP kinase or cGMP-dependent kinase, Blood 103 (2004) 2601-2609.

[37] Z. Li, X. Xi, X. Du, A mitogen-activated protein kinase-dependent signaling pathway in the activation of platelet integrin alpha IIbbeta3, J. Biol. Chem. 276 (2001) 42226-42232. 